\title{
Intraductal Drug Delivery to the Breast: Effect of Particle size and Formulation on Breast Duct and Lymph Node Retention
}

\author{
Mibin Kuruvilla Joseph ${ }^{1}$, Md Saiful Islam ${ }^{1}$, Joshua Reineke ${ }^{1}$, Michael Hildreth ${ }^{2}$, Tofuko \\ Woyengo $^{3}$, Angela Pillatzki ${ }^{4}$, Aravind Baride ${ }^{5}$ and Omathanu Perumal ${ }^{1}$ \\ ${ }^{1}$ Department of Pharmaceutical Sciences, \\ ${ }^{2}$ Department of Biology \& Microbiology, \\ ${ }^{3}$ Department of Animal Science, \\ ${ }^{4}$ Department of Veterinary Science and Biomedical Sciences, \\ South Dakota State University, Brookings, SD 57007 \\ ${ }^{5}$ Department of Chemistry, University of South Dakota, \\ Vermillion, SD 57069
}

\section{SUPPORTING INFORMATION}




\section{Materials and Methods}

\section{Crystal violet distribution in presence of PLGA formulations}

To determine if formulations blocked the ducts, crystal violet was mixed with the PLGA formulation before intraductal injections to visualize the ductal tree. After 15-30 minutes post injection, the rat was euthanized by $\mathrm{CO}_{2}$ asphyxiation and the rat was dissected to expose the mammary gland. The crystal violet distribution in the mammary gland was imaged using Sony Cybershot with Carl Zeiss lens.

\section{In vivo studies in pig}

For the pig study, the animal was anesthetized by intramuscular injection of TKX (Telazol and Xylazine at $50 \mathrm{mg} / \mathrm{ml}$ each and ketamine at $100 \mathrm{mg} / \mathrm{ml} ; 2.5 \mathrm{~m} / 50 \mathrm{~kg}$ body weight). The hair around the nipple region was removed using hair removing cream one day before starting the study. Before starting the study, alcohol swab (70\% ethanol) was used to remove the keratin plug. The PLGA formulations were dispersed in 500-1000 $\mu 1$ of PBS containing $0.05 \% \mathrm{w} / \mathrm{v}$ Tween 80 and injected into the mammary glands using $27 \mathrm{G}$ blunt needle. Consistent with the $3 \mathrm{R}$ (replacement, reduction, and refinement) principles related to animal use in research, we used one pig to test all the formulations. All seven pairs of the mammary gland were utilized for the study (Figure S1). Since the mammary glands are not connected, the chance of spill-over from one mammary gland to another is very limited. We used 2-3 teats for each formulation and the breast distribution was studied at different time points ( 2 hrs and 4 days). At the end of the treatment, the animal was euthanized using an IV injection of pentobarbital $(1 \mathrm{ml} / 10 \mathrm{lb})$. The mammary glands were excised and imaged using in vivo imager. 
Table S1. Characteristics of Polystyrene particles

\begin{tabular}{|c|c|c|c|}
\hline Particles & Particle Size (nm) & PDI & Zeta Potential (mV) \\
\hline PS 100 nm & $99.21 \pm 2.37$ & $0.10 \pm 0.03$ & $-53.3 \pm 0.90$ \\
\hline PS 500 nm & $502.5 \pm 4.85$ & $0.18 \pm 0.04$ & $-51.26 \pm 0.56$ \\
\hline PS 1000 nm & $1006 \pm 3.05$ & $0.32 \pm 0.13$ & $-48.3 \pm 0.50$ \\
\hline
\end{tabular}

Each value indicates Mean \pm S.D $(n=3)$, PS is polystyrene, PDI is polydispersity index 
Table S2. Mass balance studies for in-vitro release of dye from PLGA formulations

\begin{tabular}{|c|c|c|c|c|}
\hline Formulation & $\begin{array}{c}\text { Amount of } \\
\mathbf{C y 5 . 5} \text { in } \\
\text { formulation } \\
(\boldsymbol{\mu g})\end{array}$ & $\begin{array}{c}\text { Amount } \\
\text { Released }(\boldsymbol{\mu g})\end{array}$ & $\begin{array}{c}\text { Amount } \\
\text { Remaining }(\boldsymbol{\mu g})\end{array}$ & $\begin{array}{c}\text { Total amount } \\
\text { (released and } \\
\text { remaining in the } \\
\text { formulation) }\end{array}$ \\
\hline $\begin{array}{c}\text { PLGA } \\
\text { Microparticles }\end{array}$ & 6.34 & $1.11 \pm 0.03$ & $4.98 \pm 0.22$ & $6.09 \pm 0.19$ \\
\hline PLGA in situ gel & 6.34 & $0.56 \pm 0.18$ & $5.63 \pm 0.16$ & $6.19 \pm 0.34$ \\
\hline PLGA Nanoparticle & 3.60 & $1.53 \pm 0.05$ & $2.18 \pm 0.01$ & $3.71 \pm 0.06$ \\
\hline
\end{tabular}

Each value indicates Mean \pm S.D $(n=3)$ 


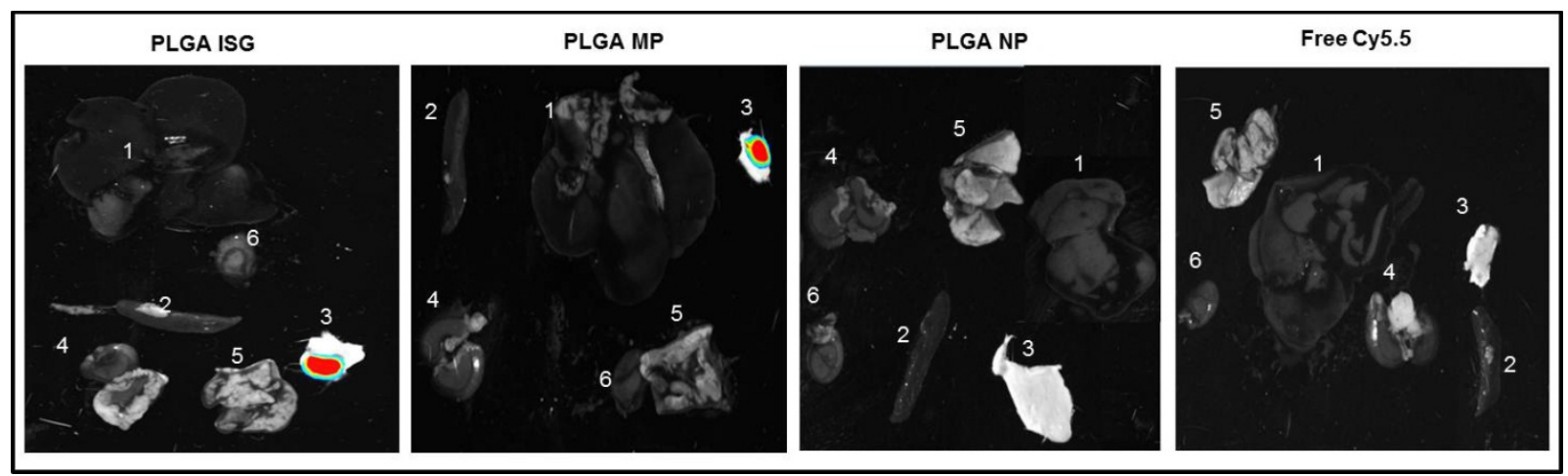

Figure S1: Representative fluorescence images showing in organ biodistribution of PLGA in situ gel (ISG), microparticles (MP), nanoparticles (NP) and free Cy5.5 after intraductal injection in rat. Organs (liver, kidney, lungs, spleen and heart) and mammary glands were excised and imaged at 60 hours for PLGA nanoparticles, 96 hours for PLGA in situ gel and microparticles, and 4 hours for free Cy5.5. 1 - liver, 2 spleen, 3- mammary gland, 4- kidneys, 5- lungs, 6- heart. 


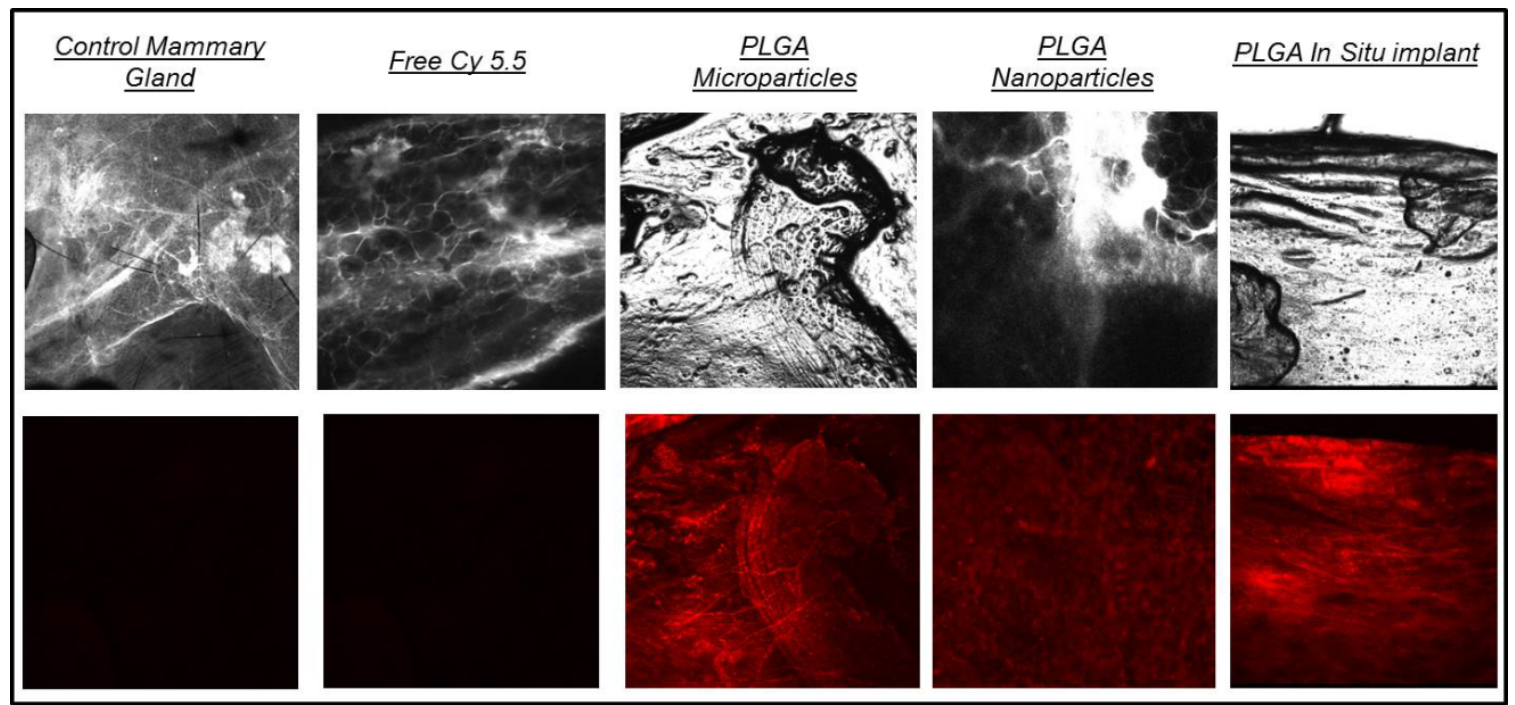

Figure S2: Mammary whole mount images after intraductal injection of free dye and PLGA formulations. The images were viewed under $20 \mathrm{X}$ using a confocal microscope 


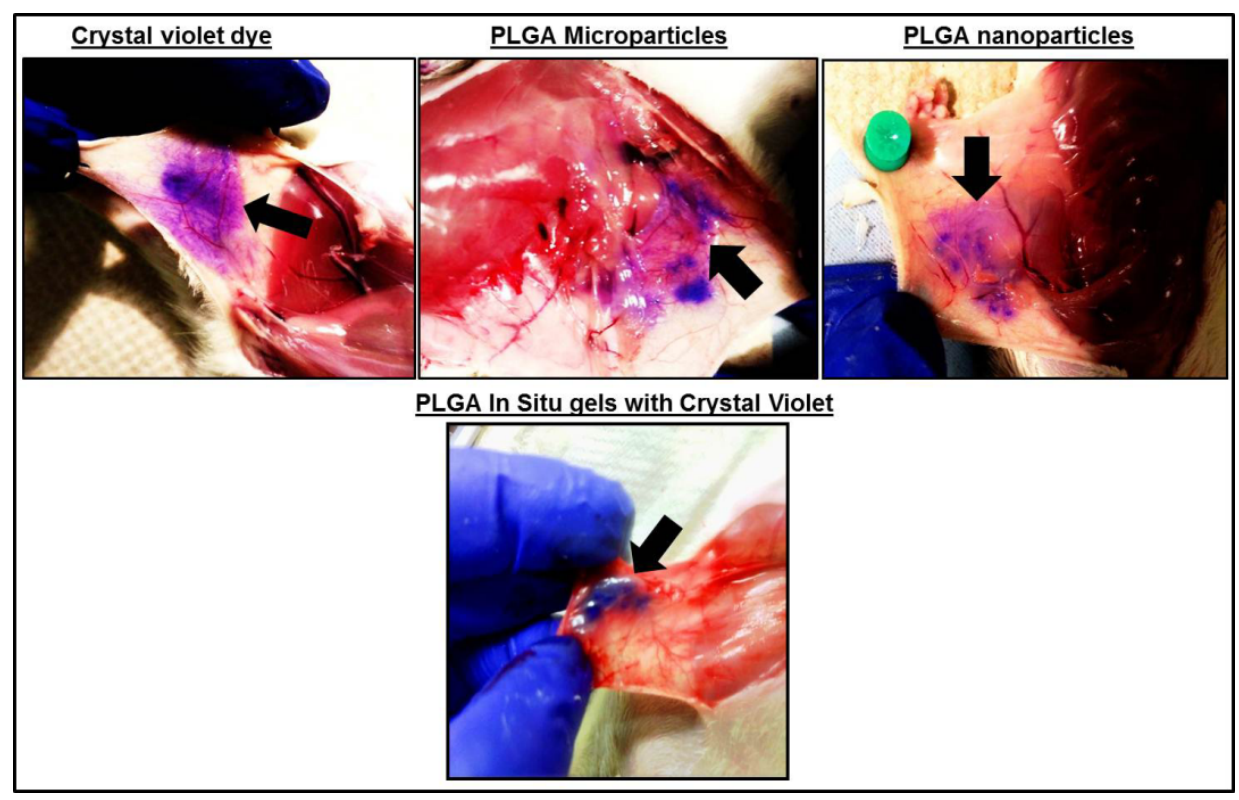

Figure S3: Intraductal distribution of crystal violet after mixing with PLGA formulations. The arrow indicates the ductal network. 


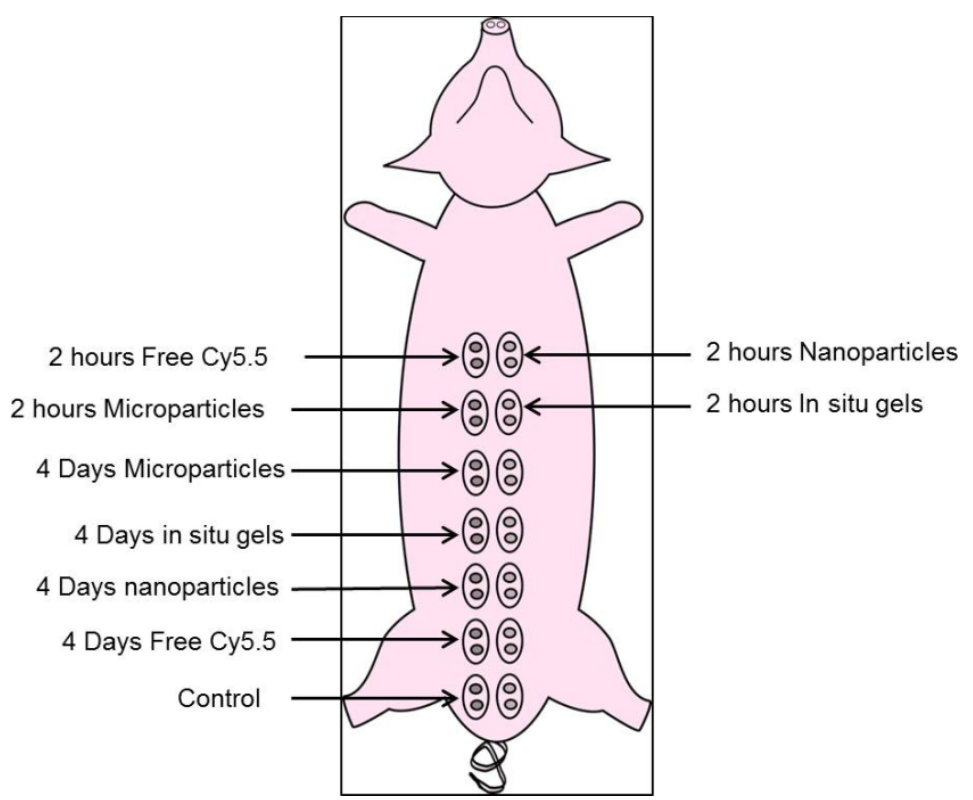

Figure S4: Schematic representation of the treatment design for PLGA formulations in pig. 


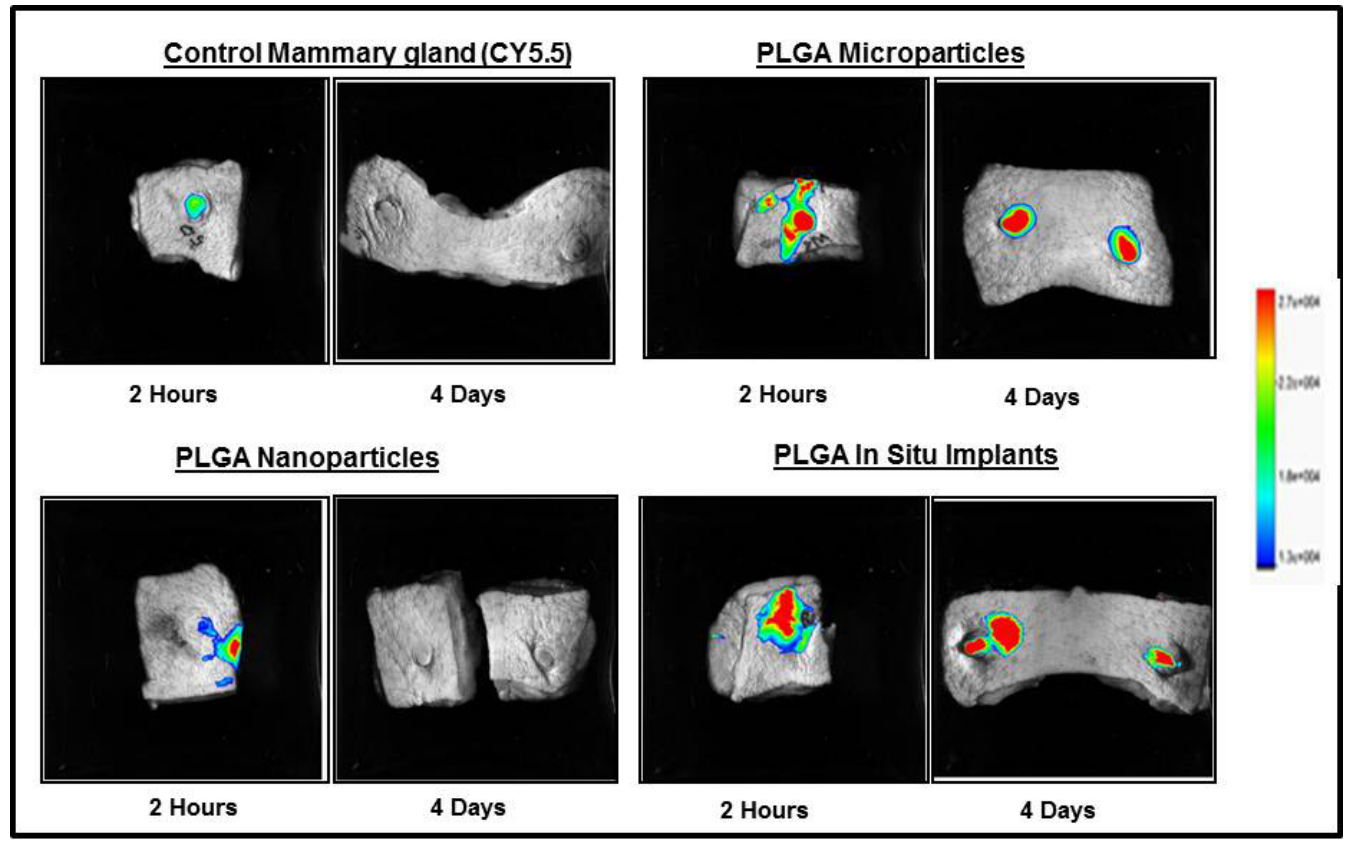

Figure S5: Representative fluorescence images (ex-vivo) of porcine mammary glands. Mammary glands were excised and imaged using in-vivo imager. 